\title{
Envases de Cartón para Líquidos como Fuente de Fibra Secundaria
}

\author{
José Turrado, Martha F. Dávalos, Francisco J. Fuentes, Alma R. Saucedo \\ Universidad de Guadalajara, Centro Universitario de Ciencias Exactas e Ingeniería, Depto. \\ de Madera, Celulosa y Papel "Ing. Karl Augustin Grellmann", CUCEI, Km 15.5 Carr. \\ 40171 Zapopán, Guadalajara - Nogales, Jal., México (e-mail: jturrado@dmcyp.cucei.udg.mx )
}

Recibido Sep. 01, 2011; Aceptado Nov. 03, 2011; Versión final recibida Dic. 12, 2011

\section{Resumen}

Se propone un método para separar la fibra de los componentes principales del envase de cartón para líquidos y para evaluar las ventajas y desventajas del empleo de esta materia prima como fuente de fibras secundarias en la fabricación de papel. La resistencia mecánica del envase postconsumo se reduce por hidratación utilizando $\mathrm{NaOH}$. Este proceso destruye los puentes de hidrógeno que unen fibra-fibra. El procedimiento permite rescatar $92 \%$ de fibra existente en el envase, por ello este material se ubica como potencial alternativa de fuente de fibra secundaria para la fabricación de papel. El material recuperado registra un alto contenido de material adhesivo (stickies), aunque es factible reducir esta cantidad a niveles que permiten su utilización en la fabricación de papel.

Palabras clave: recuperación de fibras, envase multicapas, residuos de envases, materiales adhesivos

\section{Liquid Packaging Boards as Source of Secondary Fiber}

\begin{abstract}
A new method is proposed for separating fiber material from the main components of cartonboard commonly used for storing liquids and for evaluating the advantages and disadvantages of this material as fiber sources for paper manufacture. The mechanical resistance of the cartonboard has been reduced by hydration using $\mathrm{NaOH}$ to accelerate the process. This process destroys hydrogen bonding that joint fiber-fiber. The process allows recovering $92 \%$ of the existing fiber in the carton packaging, which makes this material a potential alternative as fibber source for the manufacture of paper. The recovered material contains a high quantity of stickies, although it is possible to reduce this amount to levels that allows using the recovered fiber in paper manufacture.
\end{abstract}

Keywords: fiber recovery, multilayer packaging, packaging box residue, stickies 


\section{INTRODUCCIÓN}

En la actualidad los dos grandes líderes en ventas de envases de cartón para líquidos son las empresas Tetra Pak ${ }^{\circledR}$ y Elopak $®$; Tetra $P a k \circledR$ se ha mantenido por varios años como la compañía dominante con más del $80 \%$ del mercado mundial (Kirwan, 2005; Robertson, 2002), por lo tanto las cifras reportadas por esta empresa registradas a continuación proporcionan idea de la magnitud del impacto de este envase en el mercado mundial (Rubilar y Fuentes, 2011; Tetra Pak® group, 2011).

Tabla 1: Los ocho países más importantes en venta de envases en el 2008, (Román, 2011)

\begin{tabular}{cc}
\hline País & Millones de envases en el 2008 \\
\hline China & 27.548 \\
Brasil & 9.778 \\
Estados Unidos y Canadá & 7.810 \\
Península Ibérica & 7.505 \\
Alemania y Suecia & 6.852 \\
Japón & 6.201 \\
Rusia & 6.188 \\
México & 5.917 \\
\hline
\end{tabular}

El envase multicapas para líquidos se diseña con dos objetivos: a).- conservar el producto con refrigeración (no aséptico) y b).- conservar el producto sin refrigeración (aséptico). El envase aséptico integra una lámina de aluminio. En ambas presentaciones cada una de las láminas que le componen desarrolla una función específica, donde la lámina de cartón (Rubilar y Fuentes, 2011; Neves, 1999) es fibra celulósica y participa con $75-80 \%$ del peso total del envase, mientras que la capa de polietileno de baja densidad integra entre $15-20 \%$ del peso del mismo y la fracción de aluminio solo impacta con el $5 \%$ del peso total.

El reciclado del envase de cartón tuvo auge a partir del año 1990 con la Declaración Europea sobre la recuperación y reciclaje de papel. Por lo tanto, la necesidad de aprovechar los materiales del envase de cartón para líquidos lo ha llevado a ser objeto de diversos estudios. La tecnología actual para la reutilización de los envases de cartón para líquidos se puede agrupar en dos procesos: a) Separación de sus principales componentes (cartón, polietileno y aluminio) con la finalidad de integrar a la fibra en un proceso de papel (Bowman et al., 2007, Nieminen et al., 2002, Bouchette et al., 2002). b) Fabricación de aglomerados con toda o parte de la estructura del envase (Murathan et al., 2007; Lopes et al., 2007).

El reciclado de los envases de cartón para líquidos debe tomar en cuenta la composición de las láminas que le integran: El cartón del envase multicapa está integrado por fibras de madera de pino obtenidas por procedimientos de alto rendimiento (Kirwan, 2005; Söderhjelm y SipiläinenMalm, 1996). Las fibras de pino son fibras largas, las cuales aportan resistencia físico-mecánica al envase. Además de esta propiedad el cartón debe ser resistente a la penetración de líquidos, propiedad que se logra al bloquear las terminales activas de la fibra mediante la aplicación de resina, procedimiento que se denomina "encolado".

El polietileno proporciona a los envases hermeticidad e impermeabiliza al exterior del envase como al interior del mismo. La superficie en contacto con el alimento será siempre polietileno de baja densidad. Esta capa en contacto con el aluminio puede ser de un polietileno especial, dependiendo de la naturaleza del producto, es decir, en función del pH del líquido envasado (p. ej. aceite vegetal) (Kirwan, 2005).

La estructura del envase aséptico incluye una hoja fina de aluminio, la cual sirve como barrera contra la penetración de gases desde el exterior (oxígeno, componentes aromáticos volátiles), esta también evita que componentes aromatizantes del propio producto se pierdan. Además sirve como conductor de un potente impulso de corriente eléctrica inductiva en el momento de sellar el envase de forma transversal (Kirwan, 2005). 
El proceso de separación de fibras varía de acuerdo al tipo y cantidad de contaminantes a remover. Por lo que para no afectar negativamente las características de la fibra se necesita una clara idea de los procedimientos en la recuperación de fibra a partir del envase de cartón. La resistencia mecánica del envase puede ser reducida por hidratación o utilizando un acelerador de este fenómeno como $\mathrm{NaOH}$, este proceso destruye los puentes de hidrógeno que unen fibra-fibra (Holik, 2000). Ali y Nystrom, (1995) desarrollaron un proceso utilizando un hidrapulper, en el cual por acción mecánica se destruye el envase liberando fibras. Las condiciones de trabajo reportadas son: Temperatura $35-75^{\circ} \mathrm{C}$, tiempo de $30-45 \mathrm{~min}$ y $\mathrm{pH}$ entre $8-10$; los autores no reportan rendimiento ni consistencia de operación y concluyeron que la fibra obtenida estaba muy contaminada por partículas plásticas y metálicas.

El envase de cartón para líquidos en su etapa postconsumo es un problema ecológico, ya que forma parte del residuo sólido urbano, este material por su estructura es a su vez una fuente potencial de materias primas fibrosas por el cartón que lo constituye, el cual consta de fibras vegetales con posibilidades de ser recuperadas para ser nuevamente integradas en una nueva formulación de papel. El presente trabajo se desarrolló con el objetivo de separar la fibra de los componentes principales del envase de cartón fibra y evaluar las ventajas y desventajas del empleo de esta materia prima como fuente de fibras secundarias.

\section{METODOLOGÍA}

En el mercado se comercializa gran variedad de productos líquidos y semilíquidos en envases flexibles base cartón en diferentes presentaciones. La selección de los envases de cartón para líquidos en este estudio consideró su disponibilidad en el mercado local. El proceso de recuperación de fibra se realizó utilizando un molino de bolas de acero inoxidable, el cual fue fabricado en la Universidad de Guadalajara, las características de este molino son: Capacidad, $35 \mathrm{~L}$, longitud $0,85 \mathrm{~m}$, diámetro $0,32 \mathrm{~m}$; se utilizaron 32 cuerpos moledores (bolas) de acero inoxidable con $2,54 \mathrm{~cm}$ de diámetro y pesa cada uno en promedio $95 \mathrm{~g}$. El procedimiento para la separación de los componentes del envase de cartón fue analizado en función del comportamiento de los siguientes parámetros: "tiempo de desintegración, consistencia (relación

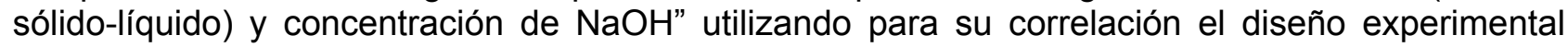
"método simplex" (Blechshmidt et al., 1979). El método simplex parte de una figura geométrica de $\mathrm{n}$ dimensiones constituida de $\mathrm{n}+1$ puntos. Cada dimensión corresponde a una variable a ser optimizada.

El método simplex en dos dimensiones es un triángulo en tres dimensiones es un tetraedro y así sucesivamente. Los parámetros y rangos de trabajo en el presente estudio se muestran a continuación: a: Tiempo de residencia, min: 20 - 37; b: Consistencia. \%: 2,97 - 4,6; c: Concentración de $\mathrm{NaOH}, \%: 0,1-0,3$.

Este método establece la matriz de operación (Blechshmidt et al., 1979):

$$
D=\left[\begin{array}{ccccc}
x_{1} & x_{2} \cdots & x_{j} \cdots & x_{n-1} & x_{n} \\
-x_{1} & x_{2} \cdots & x_{j} \cdots & x_{n-1} & x_{n} \\
0 & -2 x_{2} \cdots & x_{j} \cdots & x_{n-1} & x_{n}
\end{array}\right]
$$

Donde:

$$
x_{j}=\sqrt{\frac{1}{2_{j}(j+1)}}
$$

La materia prima seleccionada para el presente estudio en función de su presentación se registran como Envase A, B, C, D, E y F. La composición porcentual de los materiales componentes de los envases seleccionados se registra en la Tabla 2. 
Tabla 2: Composición porcentual de las principales capas que integran el envase

\begin{tabular}{cccccc}
\hline Tipo de & & \multicolumn{4}{c}{ Componentes } \\
\cline { 3 - 6 } envase & Gramaje, $\mathrm{g} / \mathrm{m}^{2}$ & \multicolumn{2}{c}{ Cartón } & \multicolumn{2}{c}{ Material no celulósico } \\
\cline { 3 - 6 } & & Ceniza, \% & Fibra, \% & Plástico, \% & Aluminio, \% \\
A & 459,7 & 1,2 & 74,0 & 19,5 & 6,5 \\
B & 433,3 & 1,2 & 74,0 & 19,5 & 6,5 \\
C & 119,0 & 1,4 & 74,0 & 19,5 & 6,5 \\
D & 315,9 & 1,4 & 74,0 & 19,5 & 6,5 \\
E & 328,3 & 1,4 & 71,5 & 23,1 & 5,4 \\
F & 464,4 & 1,4 & 71,5 & 23,1 & 5,4 \\
\hline
\end{tabular}

Las muestras seleccionadas se lavaron y se abrieron en forma longitudinal Fig. 1 para incrementar la superficie de impacto de las bolas del molino utilizado, en las imágenes se muestra el estado de los envases antes de ser sometido al proceso físico-químico de obtención de fibras.

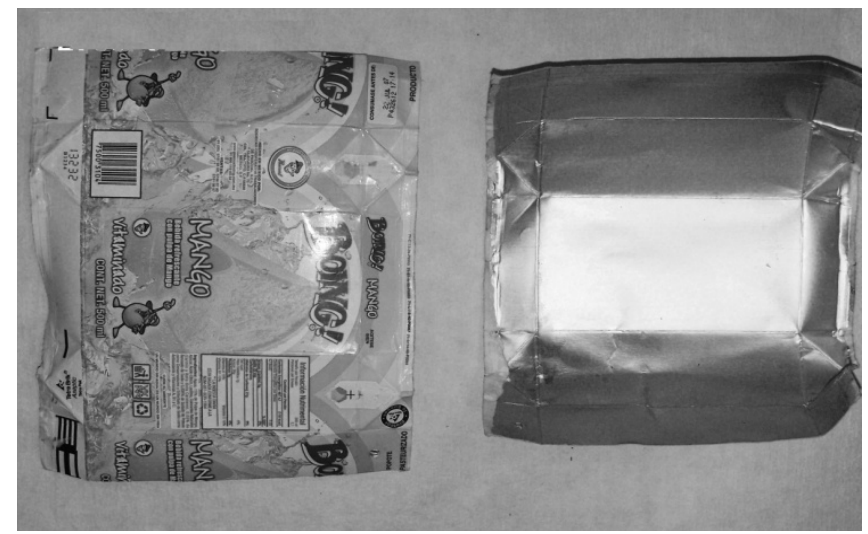

Fig. 1: Envases de cartón para líquidos extendidos

De acuerdo al diseño experimental empleado y los parámetros de estudio determinados, los parámetros seleccionados para la experimentación fueron: Consistencia 2,8 - 4,6\%, concentración de $\mathrm{NaOH}$ 0,18 - 0,34\% y tiempo de tratamiento, rango de operación; 25 - 38min, se muestra en la Tabla 3 las condiciones experimentales calculadas con el método simplex.

Tabla 3: Datos experimentales calculados y cantidad de fibra en el proceso de la recuperación de la fibra del envase de cartón para líquidos

\begin{tabular}{ccccccc}
\hline $\begin{array}{c}\text { Tiempo, } \\
\text { min }\end{array}$ & $\begin{array}{c}\text { Consistencia, } \\
\%\end{array}$ & $\begin{array}{c}\mathrm{NaOH}, \\
\%\end{array}$ & $\begin{array}{c}\mathrm{W}_{\text {envases entrada }} \\
\text { molino, }\end{array}$ & $\begin{array}{c}\mathrm{W}_{\text {fibra b.s, }}, \\
\mathrm{g}\end{array}$ & $\begin{array}{c}\mathrm{W}_{\text {fibra recuperada, }} \\
\mathrm{g}\end{array}$ & $\begin{array}{c}\text { Rendimiento, } \\
\%\end{array}$ \\
\hline 27,5 & 4,6 & 0,34 & 281,2 & 188,6 & 116,0 & 61,5 \\
22,5 & 4,6 & 0,34 & 281,2 & 188,6 & 108,0 & 57,3 \\
25,0 & 2,8 & 0,34 & 171,2 & 114,8 & 73,0 & 63,6 \\
25,0 & 4,0 & 0,18 & 244,5 & 164,0 & 115,6 & 70,5 \\
29,2 & 3,0 & 0,23 & 183,4 & 123,0 & 84,7 & 68,9 \\
29,4 & 3,0 & 0,43 & 183,4 & 123,0 & 66,0 & 53,6 \\
38,0 & 4,6 & 0,34 & 281,2 & 188,6 & 173,6 & 92,0 \\
\hline
\end{tabular}

La cantidad de materia prima ( $\left.\mathrm{W}_{\text {envase entrada molino }}\right)$ se calculó a partir de la ecuación (3), la cual define la consistencia $(C, \%)$ como la relación entre una masa $\left(X_{\text {sólidos }}\right)$ y un volumen de $100 \mathrm{~mL}$.

$$
C,(\%)=\frac{X_{\text {sólidos }}}{100(\mathrm{~mL})} \times 100
$$

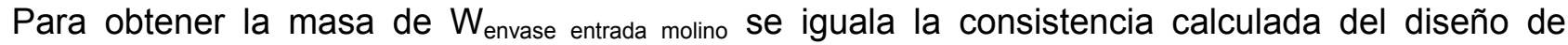
experimentos $\left(\mathrm{C}_{\text {diseño }}(\%)\right)$ y consistencia calculada para el molino $\left(\mathrm{C}_{\text {molino }}(\%)\right)$ en la ecuación (4). 


$$
C_{\text {diaseño }},(\%)=C_{\text {molino(\%) })} \frac{X_{\text {sólidos }}(g)}{100(\mathrm{~mL})} \times 100=\frac{X_{\text {envases }}(g)}{6.113(\mathrm{~mL})} \times 100
$$

Donde $6.113 \mathrm{~mL}$ es el volumen óptimo determinado experimentalmente para trabajar en el molino de bolas, al despejar $\mathrm{X}_{\text {envase }}$ se obtiene la ecuación 5.

$$
\mathrm{X}_{\text {envases }}(\mathrm{g})=6.113(\mathrm{~mL}) \times \frac{\mathrm{X}_{\text {sólidos }}(\mathrm{g})}{100(\mathrm{~mL})}
$$

Los valores del rendimiento (\%) están calculados con respecto a la $\mathrm{W}_{\text {fibra b. s. }}$ (base seca). El $\mathrm{W}_{\text {fibra }}$ b. s. se obtiene de sustraer la humedad $6,2 \%$ del peso del cartón en el envase para líquidos.

El proceso de desintegración se realizó de acuerdo a las condiciones establecidas por el diseño experimental seleccionado. En la Fig. 2 se muestra el material desintegrado al interior del molino de bolas. En esta figura se pueden observar las películas de aluminio y plástico desprendidas.

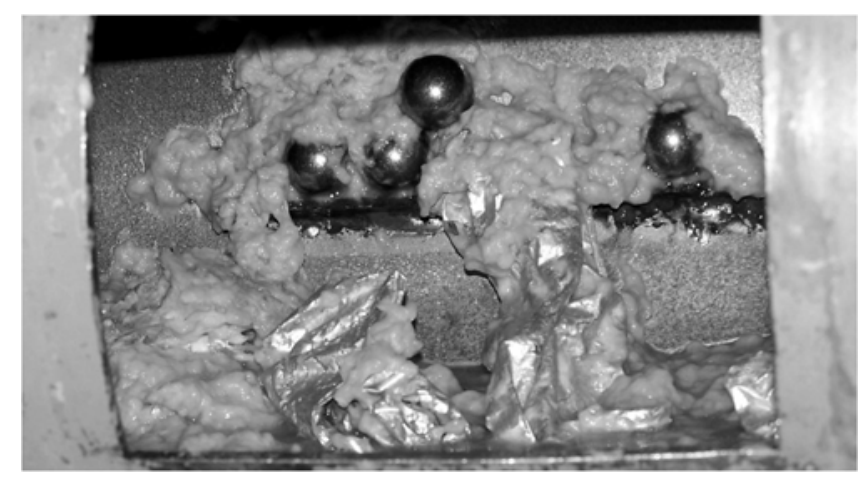

Fig. 2: Interior del molino de bolas

\section{RESULTADOS Y DISCUSION}

En la Tabla 4 se muestra el desglose del experimento en el que se obtuvo mayor rendimiento (Tabla 3). Al molino ingresan $281,2 \mathrm{~g}$ de envases de cartón para líquidos, considerando la información de la Tabla 2, la fracción de fibra en base al peso fue de 201,1g [71,5\%], mientras que el material no celulósico (aluminio + plástico) representa $80,1 \mathrm{~g}[28,4 \%]$. La fibra recuperada con respecto a la fibra b.s. fue de $92,0 \%$ de rendimiento.

Tabla 4: Cantidad presente de los principales componentes de la materia prima empleada

\begin{tabular}{cccccccc}
\hline Condiciones experimentales & $\begin{array}{c}\mathrm{W}_{\text {envases }} \\
\text { entrada } \\
\text { molino, }\end{array}$ & $\begin{array}{c}\mathrm{W}_{\text {fibra-h, }} \\
\mathrm{g}\end{array}$ & $\begin{array}{c}\mathrm{W}_{\text {aluminio+ }} \\
\text { plástico, }\end{array}$ & $\begin{array}{c}\mathrm{W}_{\text {fibra }} \\
\text { b.s, },\end{array}$ & $\begin{array}{c}\mathrm{W}_{\text {fibra }} \\
\text { recuperada, } \\
\mathrm{g}\end{array}$ & $\begin{array}{c}\mathrm{W}_{\text {aluminio+ }} \\
\text { plástico } \\
\text { obtenido, } \\
\mathrm{g}\end{array}$ & $\begin{array}{c}\text { Rendimiento, } \\
\%\end{array}$ \\
\hline 38min, 4,6 C\%, 0,34\%NaOH & 281,2 & 201,1 & 80,1 & 187,8 & 173,6 & 74,5 & 92,0 \\
\hline $\begin{array}{c}\text { Participación porcentual de } \\
\text { material, \% }\end{array}$ & 100,0 & 71,5 & 28,5 & & & & \\
\hline
\end{tabular}

En la Tabla 5 se presentan las características generales del material (envases de cartón para líquidos) previo al proceso de separación de la fibra. Estos valores representan el promedio de los datos reportados para los envases $\mathrm{E}$ y $\mathrm{F}$ (Tabla 2). Considerando que el cartón para líquidos se fabrica en diferentes partes del mundo, esté registra ligeras diferencias, así los resultados del presente trabajo tienen concordancia con los reportados por Abreu (2002): El cartón participa con $75-80 \%$ del peso total, mientras que polietileno de baja densidad integra entre $15-20 \%$ y la fracción de aluminio alcanza el $5 \%$. 
Tabla 5: Características determinadas experimentalmente para los envases recolectados

\begin{tabular}{ccccc}
\hline \multirow{2}{*}{ Gramaje, $\mathrm{g} / \mathrm{m}^{2}$} & \multirow{2}{*}{ Ceniza, $\%$} & \multicolumn{3}{c}{ Componentes, $\%$} \\
\cline { 3 - 5 } & 1,4 & Fibra & Plástico & Aluminio \\
\hline 396,3 & 71,5 & 23,1 & 5,4 \\
\hline
\end{tabular}

La Tabla 6 muestra los valores promedio obtenidos al evaluar el valor de retención de fibra método Bauer McNett de acuerdo a T233-cm-95, la fibra en el envase de cartón antes de entrar al molino presenta $53,9 \%$ de fibra retenida en la malla\# 30 (fibra larga), comparada con otras fuentes de papel reciclable para esta misma malla, el papel de revista presenta 15,0\% y el papel periódico $25,0 \%$, Castillo (2007). La fibra recuperada mediante las condiciones experimentales $38 \mathrm{~min}$, $4,6 \% \mathrm{C}, 0,34 \% \mathrm{NaOH}$ presentan una retención en la malla\# 30 de $41,8 \%$, lo cual indica la pérdida del $12,1 \%$ de fibra larga respecto al valor de la fibra en el envase antes de entrar al molino. El impacto del proceso mecánico sobre las fibras recuperadas se aprecia en la cantidad de finos reportados en la Tabla 6.

Tabla 6: Clasificación de fibras del envase con el método Bauer McNett T23-cm-95 y su comparación con los reportados por la referencia mencionada

\begin{tabular}{cccccc}
\hline \multirow{2}{*}{ Muestra } & \multicolumn{5}{c}{ Fibra retenida en malla\#, \% } \\
\cline { 2 - 6 } & 30 & 50 & 100 & 200 & Finos \\
\hline Fibra sin tratamiento & 53,9 & 7,8 & 3,9 & 5,8 & 28,6 \\
Fibra recuperada & 41,8 & 10,9 & 4,2 & 6,5 & 36,5 \\
Papel revista & 14,7 & 10,0 & 5,1 & 20,7 & 49,5 \\
Papel periódico & 24,7 & 10,8 & 8,9 & 24,3 & 31,3 \\
\hline
\end{tabular}

El análisis de la calidad de la fibra (FQA) de acuerdo a la técnica T271-cm-02 en la fibra recuperada se observa en la Tabla 7 . Este análisis proporciona sus principales características como son: Largo promedio de fibra (Ln), Largo promedio ponderado de fibra (Lw); Largo promedio ponderado en peso de fibra (Lww), así como Índice de Kink (ángulos en la fibra) e Índice de Curl (curvatura de la fibra).

Tabla 7: Análisis de la fibra con el FQA (Fiber Quality Analyzer)

\begin{tabular}{|c|c|c|c|c|c|c|}
\hline \multirow[t]{2}{*}{ Muestra } & \multicolumn{2}{|c|}{ Longitud de fibra } & \multirow{2}{*}{$\begin{array}{l}\text { Finos } \\
1 / \mathrm{mm}\end{array}$} & \multicolumn{2}{|c|}{ Índice de Curl } & \multirow{2}{*}{$\begin{array}{c}\text { Índice de } \\
\text { Kink } \\
\text { Lw, \% }\end{array}$} \\
\hline & $\mathrm{Ln}, \mathrm{mm}$ & $\mathrm{Lw}, \mathrm{mm}$ & & Lww, mm & Ln, \% & \\
\hline malla\# 30 & 1,1 & 1,9 & 1,7 & 2,6 & 17,1 & 1,9 \\
\hline
\end{tabular}

La mezcla de materiales adhesivos presentes como resinas de encolado en el cartón y las resinas empleadas en la unión entre capas, se denominan "stickies". La cuantificación de materiales adhesivos presentes "microstickies" en una suspensión fibrosa se efectuó de acuerdo al procedimiento desarrollado por Turrado et al., (1999), este procedimiento se ha aplicado como apoyo a estudios sobre el tema para diferentes empresas en México, ya que no hay un procedimiento estándar. La evaluación de microstickies en la fibra del cartón previo al proceso de separación se denominó "Blanco" (Tabla 8), esta fibra se separó del envase con las manos. Así mismo se reporta el valor obtenido de la fibra recuperada del proceso estudiado en este estudio. La cantidad de microstickies representa la presencia de partículas adhesivas en un área por un kilogramo de fibra, los valores reportados para la fibra recuperada de revista estucada tomada como referencia indica un valor controlable en la máquina de papel (Turrado et al., 1999). Se aprecia en la Tabla 8 que la fibra del envase de cartón contiene el doble que en el papel revista, al separar la fibra utilizando el molino de bolas el valor de microstickies se triplica con respecto al Blanco, por lo que al emplearse la fibra del envase de cartón para líquidos en la producción de papel requerirá de un tratamiento para contrarrestar la presencia de material adhesivo en la fibra. 
Tabla 8: Cantidad de microstickies en la fibra

\begin{tabular}{cc}
\hline Experimento & $\mathrm{cm}^{2} / \mathrm{Kg}$ \\
\hline Blanco & 153 \\
Fibra recuperada & 467 \\
Revista estucada & 61 \\
\hline
\end{tabular}

El procedimiento desarrollado en la Universidad de Guadalajara detecta la presencia de partículas en función de su tamaño $\left(>2 \mu \mathrm{m}^{2}\right)$, se graficó la distribución del número de tamaño de partículas por rango de tamaño lo cual se muestra en la Fig. 3. La gráfica muestra la disminución de la población de partículas a partir del área mínima seleccionada de $2 \mu \mathrm{m}^{2}$ hasta la superficie creciente de aproximadamente $292 \mu \mathrm{m}^{2}$.

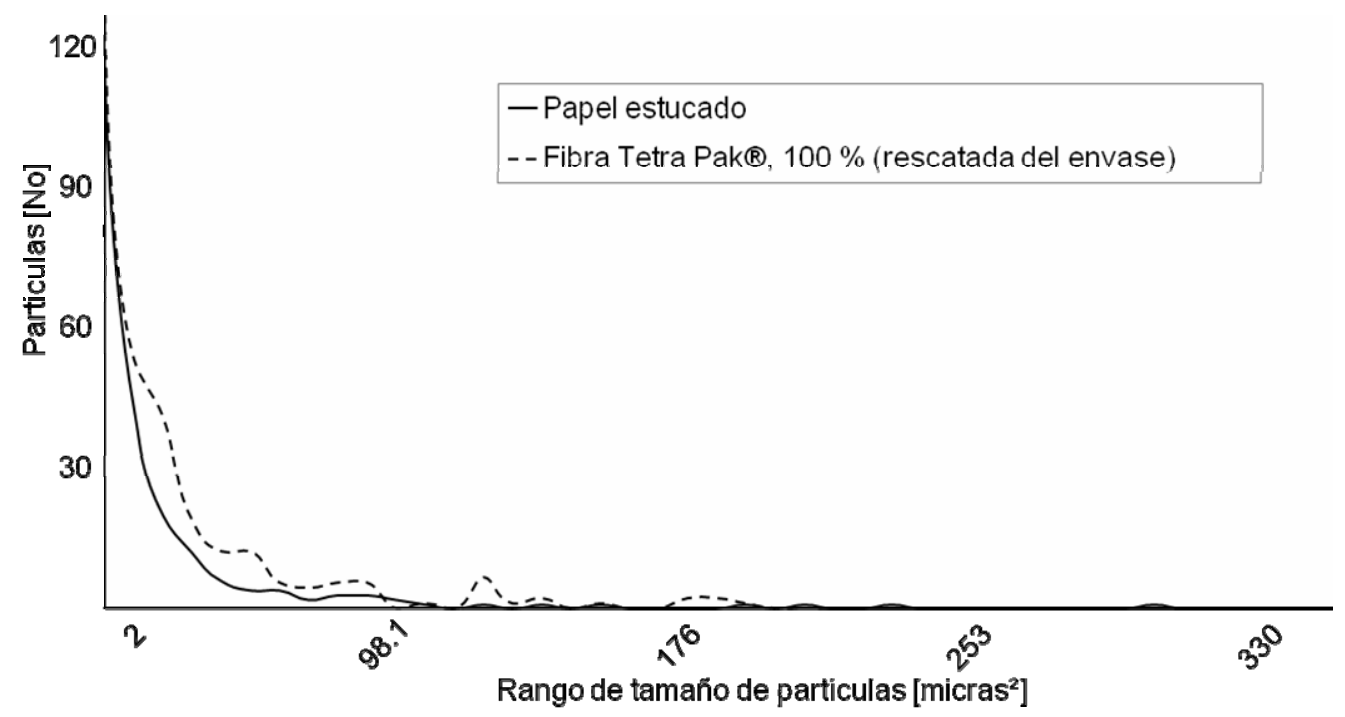

Fig. 3: Gráfica de las partículas detectadas de micro-stickies en el cartón del envase multicapas

\section{CONCLUSIONES}

El procedimiento de este estudio permite rescatar el $90 \%$ de la fibra celulósica presente en el envase. El material fibroso recuperado registra en malla\# 30 una retención del $54 \%$, lo que permite ubicar este material como potencial alternativa de fuente de fibra secundaria para la fabricación de papel.

El material recuperado registra un alto contenido de stickies $467 \mathrm{~cm}^{2} / \mathrm{Kg}$ sin embargo es factible reducir esta cantidad a niveles que permiten su utilización en la fabricación de papel.

\section{REFERENCIAS}

Abreu M., Reciclagem de embalagens cartonadas Tetra Pak para alimentos líquidos, O Papel: 64 , 91-96 (2002).

Ali, H. y T. Nystrom, Method and apparatus for separating paper fiber and plastics from mixed wasted material and products obtained thereby, United States -Patent Number 5,390,860, 1-10 (1995).

Blechshmidt, J., Chr. Bäurich y J. Papp, Anwendung der Simplex-Methode zur Optimierung der Zugfestigkeit bei der Aufbereitung nafester Altpapiere, Zellsoff 1, und Papier: 3, 136-138 (1979). 
Bouchette M.P., W.F. Winkler y H.C. Thomas, Method for removing contaminants from fibers in recycle fiber pulping. United Satated patent application publication: No: US 2002/0096269 A1, 1-25 (2002).

Bowman D.J., T. R. Eason y J. W. Renz, Apparatus for liquid-based fiber separation. United States patent No: US7,279,073 B2, 1-21 (2007).

Castillo L., Estudio de los mecanismos fisicoquímicos en el proceso de destintado por flotación de papel desperdicio. Tesis de Maestría, Universidad de Guadalajara, Departamento de Celulosa, Madera y Papel, Guadalajara, México (2007).

Holik, H., Units operations and equipment in recycled fiber processing. Recycled fiber and deinking, $1^{\text {a }}$ edición, Fapet Oy, vol 7, 90-209, Helsinki, Finlandia (2000).

Kirwan, J.M., Paperboard-based liquid packaging. Paper and paperboard packaging technology, 386-413, Blackbewell Publishing, Oxford, Inglaterra (2007).

Lopes M., M. Gonçalves y M. Felisberti, Blends of poly(ethylene terephthalate) and low density polyethylenecontaining aluminium: A material obtained from packaging recycling. Journal of Applied polymer science: 106, 2524-2535 (2007).

Murathan, A., S. Murathan, M. Gürü y M. Balbaşi, Manufacturing low density boards from waste cardboards cointaining aluminium. Materials and design: 28, 2215-2217 (2007).

Neves F.L."Reciclagem de Embalagens Cartonadas Tetra Pak", O Papel: 61 (2) , 38-41 (1999).

Nieminen J., J. Palonen, M. Itäpelto, P. Harkki y L. Mäipaja, Liquid carton waste material recycling process and apparatus for recycling liquid carton waste material. United States patent No: US 6,402,635 B1, 1-7 (2002).

Robertson, G., The paper beverage carton: Past and future. Foodtechnology: 7(56), 46-52 (2002).

Román, A., Tecnologías ambientales de producto y proceso - Tetra Pak® México. Foro nacional de formación. Innovación y mejora de sistemas, productos, procesos y servicios. Toluca, México 26 y 27 de Noviembre del 2009 http://www.ctteb.com/Foro-Nacional/index.html. Acceso: 18 de marzo (2011).

Rubilar C. y O. Fuentes, Nuevas posibilidades para el reciclaje de envases multicapa, Ecoamerica http://www.ecoamerica.cl/sitio/index.php?area=163. Acceso: 15 de Mayo (2011).

Söderhjelm, L. y T. Sipiläinen-Malm, Paper and board, Migration from food contact materials, $1^{\text {a }}$ edición, Blackie Academic and Professional, 159 -162, Londres, Inglaterra (1996).

Tetra Pak® group, "Tetra Pak forecasts continued growth in global milk consumption over next three years." ProQuest Newsstand. Document ID: 1755040631 http://proquest.umi.com/pqdweb?did=1755040631\&sid=1\&Fmt=3\&clientld=31312\&RQT=309\&VNa me=PQD. Acceso: 12 de Noviembre (2011).

Turrado, J., O. González, C. Vázquez, A. R. Saucedo y S. Pérez, Contaminant (stickies) Content Procedure, Progress in Paper Recycling: 8, 80-82 (1999). 